\title{
Cryo-EM Studies of Respiratory Complexes in a Hyperthermophilic Archaeon Pyrococcus furiosus Suggest an Evolutionary Path to Modern-Day Complex I
}

\author{
Hongjun $\mathrm{Yu}^{1}$, Gerrit J. Schut ${ }^{2}$, Chang-Hao $\mathrm{Wu}^{2}$, Dominik K. Haja ${ }^{2}$, Michael W. Adams ${ }^{2}$ and Huilin $\mathrm{Li}^{1}$ \\ 1. Structural Biology Program, Van Andel Research Institute, Grand Rapids, Michigan, USA. \\ 2. Department of Biochemistry and Molecular Biology, University of Georgia, Athens, Georgia, USA.
}

The modern-day respiratory Complex I is a NADH-ubiquinone oxidoreductase (NUO). It is thought to have evolved as the first complex of what would become the aerobic respiratory chain, with Complex III and IV being later additions and accessary to Complex I. Complex I or NUO complexes can be further divided into NUOB of bacteria and mitochondria that use NADH as the electron donor, while NUOA of the archaea use ferredoxin (Fd) as electron donor. Throughout Earth's history, the relative level of these oxidants increased as the redox state of the atmosphere became more and more oxidized due to the emergence and spread of oxygenic photosynthesis.

The ancestral respiratory complex to Complex I is proton-reducing due to the lack of oxygen. As the atmospheric oxygen level increased, the ancestral complex seems to have gradually evolved and diversified to several different respiratory complexes [1-2]. The first Complex I-like system to have evolved is the membrane-bound hydrogenase $(\mathrm{MBH})$, which uses $\mathrm{Fd}$ as electron donor and protons as electron acceptor. This share a common ancestor with the later evolving membrane-bound sulfane reductase that uses $\mathrm{Fd}$ as electron donor and polysulfide as electron acceptor. It is thought that subsequently the methanogen respiratory complex (FPO) evolved that uses Fd (FPOF) or cofactor $\mathrm{F}_{420}$ (FPOC) as electron donor and phenazine as electron acceptor. Finally, Complex I or NUO evolved that uses either Fd (NUOA) or NADH (NUOB) as electron donor and quinone - but ultimately oxygen via complex III and Complex IV - as the electron acceptor. $\mathrm{MBH}$ is evolutionary related to the ancient Mrp $\mathrm{Na}^{+}-\mathrm{H}^{+}$antiporter and the modern-day Complex I (Fig. 1).

Very recently we determined the structure of the Pyrococcus furiosus MBH by cryo-EM [3]. MBH is encoded by a 14-gene operon (Fig. 2; top panel), where 10 of the genes have close homologs in Complex I. We inserted at the $\mathrm{N}$ terminus of MbhJ a His9 affinity tag for purification of MBH. CryoEM data collection was performed on a $300 \mathrm{kV}$ FEI Titan Krios electron microscopy with a K2 camera positioned post a GIF quantum energy filter. We obtained a 3D map of $\mathrm{MBH}$ with an overall resolution of 3.7 $\AA$. In the 3D density map, most loops and many side-chain densities were well resolved in the experimental electron density map allowing de novo model building for 2,470 out of the total 2,502 residues of the complex. Only a flexible loop region spanning 32 residues in MbhI was not as well resolved and therefore allowed for only main chain tracing in this region.

The MBH atomic model represents the first ever structure of a group-4 [NiFe]-type hydrogenase (Fig. 2, bottom panel). Like Complex I, MBH adopts an L-shaped structure with a peripheral arm and a membrane arm. The peripheral arm is the membrane-anchored hydrogenase module that is structurally similar to the quinone-binding Q-module of complex I, while membrane arm is the ion translocation module that can be divided into $\mathrm{H}^{+}$pumping unit and a $\mathrm{Na}+\mathrm{H}+$ antiporter unit. Surprisingly, we found that the $\mathrm{H}^{+}$-translocating unit is rotated $180^{\circ}$ in-membrane with respect to its counterpart in Complex I, leading to distinctive architectures for the two respiratory systems despite their largely conserved proton-pumping mechanisms. We identified a $\mathrm{Na}^{+}$-translocating unit in $\mathrm{MBH}$, which was absent in 
Complex I. The $\mathrm{Na}^{+}$-translocating unit resembles that found in the $\mathrm{Mrp} \mathrm{H}^{+} / \mathrm{Na}^{+}$antiporter and enables hydrogen gas evolution by $\mathrm{MBH}$ to establish a $\mathrm{Na}^{+}$gradient for ATP synthesis near $100^{\circ} \mathrm{C}$.

\section{References:}

[1] GJ Schut et al., Biochim Biophys Acta 1857 (2016), p. 958.

[2] GJ Schut et al., FEMS Microbiology Reviews 37 (2013), p. 182.

[3] H Yu et al., Cell 173 (2018), p. 1636.
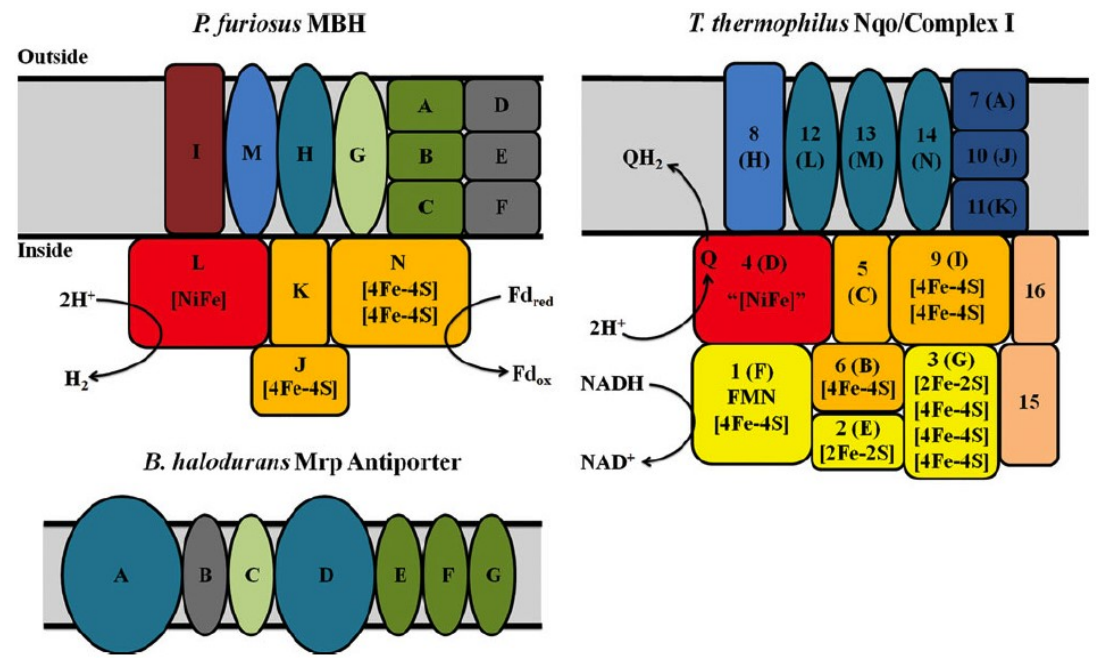

Figure 1. Membrane-bound hydrogenase $(\mathrm{MBH})$ catalyzes $\mathrm{H}_{2}$ production using reduced ferredoxin generated from sugar oxidation, as the electron donor. $\mathrm{MBH}$ is an evolutionary ancestor of modern-day respiratory Complex I. Mrp is the likely ancestor of the membrane arms of MBH and Complex I.

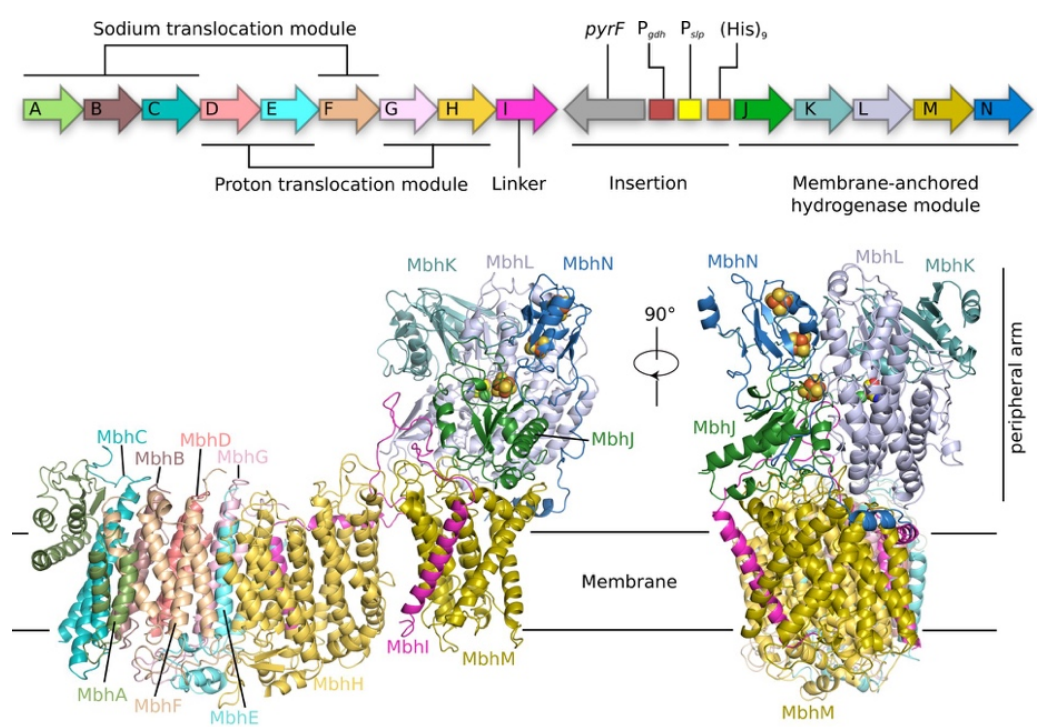

Figure 2. Top: Operon encoding the 14-protein $\mathrm{MBH}$ complex. Bottom: two orthogonal views of the MBH structure in cartoon display. Subunits are colored individually. Adapted from Yu et al. Cell, 1636$1649,2018$. 